\title{
Efeito do treinamento com flauta doce na força muscular respiratória de idosas: estudo piloto
}

\author{
Effect of sweet flute training on the respiratory \\ muscle force of elderly - a pilot study
}

Resumo Objetivo: Avaliar o efeito do treinamento com flauta doce sobre a força muscular de idosas. Métodos: Estudo quasiexperimental realizado com cinco idosas $(67 \pm 7)$. As idosas foram submetidas a oito semanas de treinamento presenciais (oito sessões) de 90 minutos com flauta doce referente à música "Noite Feliz". A mensuração da força muscular respiratória foi realizada antes e após o período de treinamento, por meio de um manovacuômetro, pela medida das pressões respiratórias máximas. Utilizou-se a estatística descritiva e inferencial, considerando p $<0,05$. Resultados: A pressão inspiratória máxima (PImáx) e a pressão expiratória máxima (PEmáx) apresentaram aumentos significantemente, após o treinamento ( $54 \pm 20$ vs $98 \pm 8 \mathrm{cmH} 2 \mathrm{O}, \mathrm{p}<0,04$ e $40 \pm 14$ vs $68 \pm 17 \mathrm{cmH} 2 \mathrm{O}, \mathrm{p}<0,03$ respectivamente). Conclusão: Houve aumento da força muscular respiratório de idosas submetidas a um treinamento com flauta doce.

Palavras-chave: Envelhecimento. Música. Trabalho respiratório. Promoção da SAÚde.

Abstract Objective: To evaluate the effect of flute training on the muscular strength of elderly women. Methods: Quasi-experimental study with five elderly women $(67 \pm 7)$. The elderly were submitted to eight weeks of face-to-face training (eight sessions) of 90 minutes with a flute referring to the song "Happy Night". Measurement of respiratory muscle strength was performed before and after the training period, using a manovacuometer, for the measurement of maximal respiratory pressures. Descriptive and inferential statistics were used, considering $\mathrm{p}<0.05$. Re-

Dayane Aparecida Viana Daniel Vicentini de Oliveira Maycon Sousa Pegorari ${ }^{\text {II }}$ Lilane Maria Alves Silva GUalberto RuAs ${ }^{\text {III }}$

LisLei Jorge Patrizzi ${ }^{\text {IV }}$

Jurema Ribeiro Luiz Gonçalves ${ }^{v}$ ${ }^{\mathrm{I}}$ Universidade Estadual de Campinas (UNICAMP). Campinas/SP - Brasil. "Universidade Federal do Triângulo Mineiro (UFTM). Uberaba/MG Brasil.

"IIUniversidade Federal de São Carlos (UFSCAR). São Carlos/SP - Brasil.

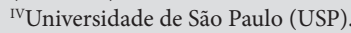

São Paulo/SP - Brasil.

vUniversidade de São Paulo (EERP-USP). Ribeirão Preto/SP - Brasil. sults: The maximal inspiratory pressure (MIP) and maximal expiratory pressure (MEP) increased significantly after training (54 \pm 20 vs $98 \pm 8$ $\mathrm{cmH} 2 \mathrm{O}, \mathrm{p}<0.04$ and $40 \pm 14$ vs $68 \pm 17 \mathrm{cmH} 2 \mathrm{O}, \mathrm{p}<0.03$ respectively). Conclusion: There was an increase in the respiratory muscle strength of elderly women undergoing flute training.

Keywords: Aging. Music. Respiratory Work. Health PromoTION.

\section{INTRODUÇão}

Para se tocar alguns instrumentos musicais de sopro, faz-se necessário ter uma preservada força muscular respiratória (FMR), assim como, a função pulmonar, para que 
o som seja produzido adequadamente. ${ }^{1}$ A flauta é um instrumento que exige resistência ao fluxo de ar, conforme a necessidade de tocar sons mais agudos. ${ }^{2-3} \mathrm{O}$ uso de instrumento de sopro exige a realização de técnicas dependentes de esforço da musculatura respiratória. ${ }^{4}$

Estudos têm demonstrado melhor FMR e função pulmonar entre profissionais musicistas que utilizam instrumentos de sopro. ${ }^{1,4}$ $\mathrm{Na}$ literatura, apenas existem estudos que abordam o uso da flauta doce como recurso da musicoterapia para fins lúdicos e melhora da sociabilidade e qualidade de vida em idosos. ${ }^{3}$ Além disso, acredita-se que possa repercutir também positivamente para o incremento ou manutenção da força muscular respiratória, tendo em vista as alterações do envelhecimento na função pulmonar. ${ }^{1}$

Cabe considerar que, devido à grande exposição a poluentes ambientais, no decorrer dos anos, o sistema respiratório é considerado o que envelhece mais rapidamente no organismo. ${ }^{5}$ Além disso, com o avançar da idade as propriedades elásticas dos tecidos dos pulmões e da parede torácica se alteram, fazendo que haja um enrijecimento dessa parede, ocasionando a diminuição da massa e eficiência dos músculos respiratórios e, por conseguinte, levando à redução da complacência pulmonar que, por sua vez, aumenta o trabalho respiratório. ${ }^{6}$

Concernente à função pulmonar, um estudo apontou que a capacidade vital forçada (CVF) reduz aproximadamente 25\% a 40\%. ${ }^{7}$ Assim, como o volume expiratório forçado no primeiro segundo $\left(\mathrm{VEF}_{1}\right)$, a relação entre $\mathrm{VEF}_{1} / \mathrm{CVF}$ também diminui conforme aumenta a idade. ${ }^{8}$ Dessa maneira, acredita-se que a utilização de diferentes intervenções além das convencionais para a melhora da força muscular respiratória, como a flauta doce, são novas estratégias de promoção da saúde em diferentes espaços e contextos. Portanto, o objetivo deste estudo foi avaliar o efeito do treinamento com flauta doce sobre a força muscular de idosas.

\section{Métodos}

Trata-se de um estudo quasiexperimental com cinco idosas alunas da Universidade Aberta à Terceira Idade (UNATI) da Universidade Federal do Triângulo Mineiro. Os critérios de inclusão envolveram idosas sem alterações significativas na função pulmonar, não fumantes e sem experiências prévias com instrumento de sopro. O estudo foi realizado após a aprovação do Comitê de Ética em Pesquisa da Universidade Federal do Triângulo Mineiro, sob protocolo $\mathrm{n}^{\circ}$. 2.307.

A CVF, $\mathrm{VEF}_{1}$ e a relação entre $\mathrm{VEF}_{1} /$ CVF foram avaliados por meio do espirômetro modelo Master Scope da Jäeger (Wuerzburga). Foram consideradas pelo menos três curvas aceitáveis de cada uma das manobras e os valores espirométricos foram expressos em porcentagem do valor predito para a população brasileira. Essa avaliação foi realizada apenas para detectar algum grau de disfunção prévia. ${ }^{9}$ A mensuração da FMR foi feita antes e após o período de treinamento. Ocorreu por meio de um manovacuômetro analógico (Ger-Ar', São Paulo, Brasil), com escalas de - $300 \mathrm{a}+300 \mathrm{cmH}_{2} \mathrm{O}$ (devidamente calibrado), equipado com traqueia de plástico de $16 \mathrm{~cm}$ de comprimento e $2,4 \mathrm{~cm}$ de diâmetro interno, bocal de plástico rígido e orifício de fuga de aproximadamente 2 $\mathrm{mm}$ de diâmetro para dissipação das pres- 
sões geradas pela musculatura da face e da orofaringe. Foram obtidas as medidas de pressão inspiratória máxima $\left(\mathrm{PI}_{\text {máx }}\right.$ e pressão expiratória máxima $\left(\mathrm{PE}_{\text {máx }}\right.$, realizadas no máximo cinco vezes, aceitáveis e reprodutíveis, sendo na posição sentada, com uso de clipe nasal, por um tempo de sustentação de três segundos. $\mathrm{O}$ valor da $\mathrm{PI}_{\text {máx }}$ foi obtido a partir do volume residual (VR), e a $\mathrm{PE}_{\text {maxx }}$, a partir da capacidade pulmonar total (CPT). $\mathrm{O}$ idoso recebeu o devido incentivo verbal e, caso houvesse diferença maior que $10 \%$ entre uma medida e outra, uma nova manobra era feita, considerando-se o maior valor obtido, desde que esse não fosse o último. ${ }^{10}$

As voluntárias foram submetidas a oito semanas de treinamento com flauta doce, uma vez por semana, totalizando oito encontros presenciais de 90 minutos. O treinamento foi oferecido por um profissional habilitado. O profissional incentivou as idosas a treinarem em casa por, no mínimo, uma hora por dia. No primeiro encontro, foram oferecidas noções básicas sobre música e flauta doce. As participantes foram orientadas a realizar treinamento com a flauta doce durante as oito semanas. A partir do segundo encontro, iniciou-se o treinamento com a música "Noite Feliz - Franz Gruber", sendo que o mesmo aconteceu até o oitavo encontro, quando todas atingiram o objetivo, concluindo a música.
Para análise dos resultados, utilizou-se o programa estatístico InStat versão 3.05 (GraphPad Software Inc, San Diego, CA, EUA). As variáveis estudadas são apresentadas em seus valores médios e desvios padrão (DP). Considerando o comportamento normal das variáveis, por meio do teste de Shapiro-Wilk, foi usado o teste $t$ Student pareado para comparação das médias pré e pós intragrupo. O nível de significância analisado foi de $\mathrm{p} \leq 0,05$.

\section{RESULTADOS}

A média de idade de $67 \pm 7$ anos, média de estatura $156 \pm 1$, média de peso $69 \pm 8 \mathrm{~kg}$ e média de índice de massa corporal (IMC) de $28 \pm 5 \mathrm{~kg} / \mathrm{m}^{2}$. Observou-se no presente estudo que as voluntárias não relataram qualquer sintoma respiratório isolado ou combinado que incluísse a presença de tosse, dispneia a pequenos esforços e dor do tipo pleurítica. Além disso, a prova de função pulmonar não mostrou alteração alguma, com média de CVF, $\mathrm{VEF}_{1}, \mathrm{VEF}_{1}$ I CVF $(80 \pm 5,82 \pm 5$ e $89 \pm 6 \%$, respectivamente). No entanto, a $\mathrm{PI}_{\text {máx }}$ e a $\mathrm{PE}_{\text {máx }}$ exibiram aumentos significantemente após o treinamento $\left(54 \pm 20\right.$ vs $98 \pm 8 \mathrm{cmH}_{2} \mathrm{O}, \mathrm{p}<0,04 \mathrm{e}$ $40 \pm 14$ vs $68 \pm 17 \mathrm{cmH}_{2} \mathrm{O}, \mathrm{p}<0,03$ respectivamente, Teste $t$ Student pareado), conforme Figuras 1 e 2. 
Figura 1. Médias e desvios padrão da $\mathrm{PI}_{\text {máx }}$ pré e pós treinamento.

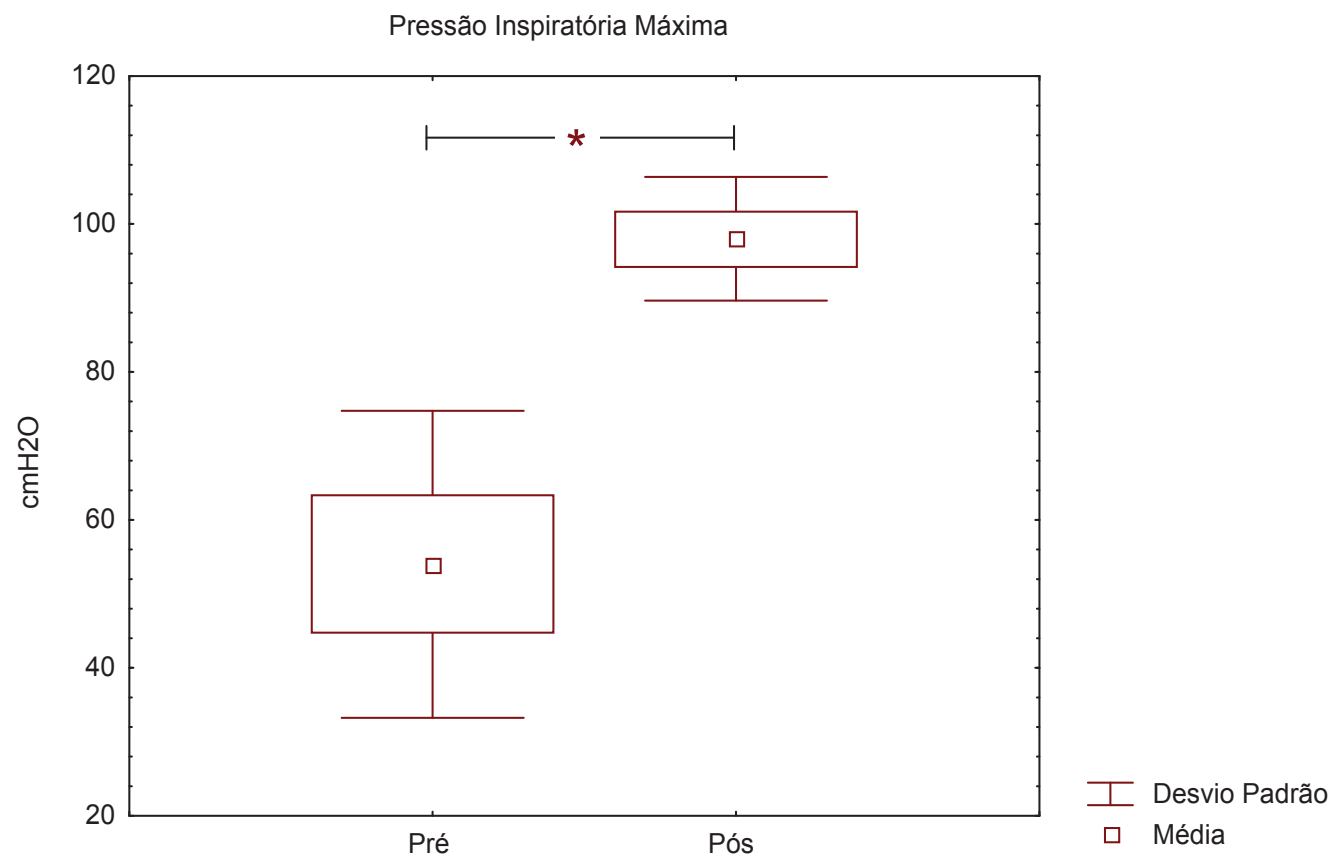

Figura 2. Médias e desvios padrão da $\mathrm{PE}_{\text {máx }}$ pré e pós treinamento

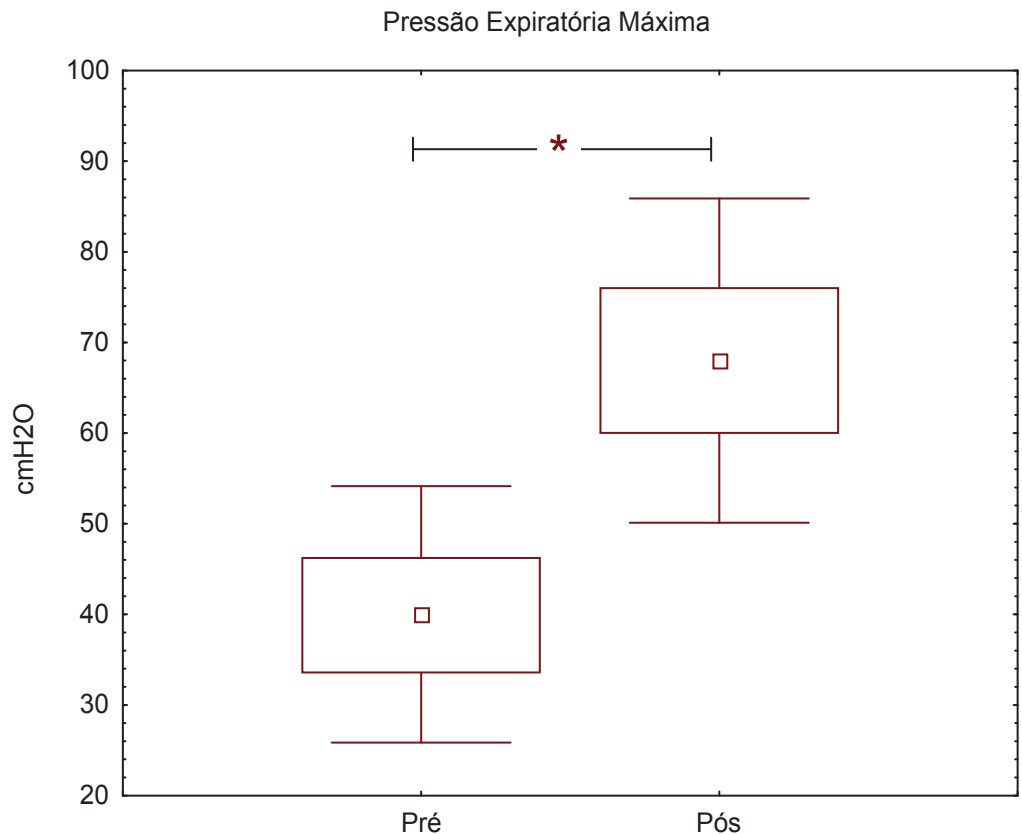

$\mp$ Desvio Padrão
$\square \quad$ Média 


\section{Discussão}

$\mathrm{O}$ estudo demonstrou que houve aumento da FMR em idosas após a execução de um protocolo de treinamento da musculatura respiratória por oito semanas com o instrumento musical de sopro flauta doce, mesmo sendo um período curto de intervenção.

Embora não tenha avaliado a população idosa, um estudo que investigou a FMR de instrumentistas que tocavam trompete em comparação com indivíduos sadios sem prática de instrumentos de sopro e com características antropométricas semelhantes obteve maiores valores de $\mathrm{PI}_{\text {máx }}$ e $\mathrm{PE}_{\text {máx }}$ entre os musicistas, identificando que os trompetistas desenvolvem pressões mais altas com seus músculos inspiratórios e expiratório. ${ }^{4}$

Tocar um instrumento de sopro demanda função ventilatória apropriada, com preservação das capacidades pulmonares para a geração de volumes adequados. Além disso, é necessário o controle preciso do fluxo de ar, assim como força dos músculos respiratórios. Como consequência, acredita-se que o fortalecimento desses músculos implique aumento da capacidade vital daqueles que tocam flauta. ${ }^{11}$ Durante a prática, impõe-se resistência à fase expiratória, ampliando a capacidade respiratória do indivíduo. ${ }^{12}$

Em contrapartida, estudo que comparou a FMR de 51 musicistas jovens e adultos praticantes de instrumentos de sopro com 26 praticantes de outros tipos de instrumentos musicais não encontrou diferenças entre os dois grupos $(p=0,331)$. Todavia, a $\mathrm{PI}_{\text {máx }}$ obtida entre aqueles pertencentes ao subgrupo praticante de tuba foi superior (-244 mmHg) em relação ao grupo contro- le (-142 $\mathrm{mmHg})$ e aos praticantes de outros instrumentos musicais de sopro, incluindo a flauta (-140 mmHg). Atribuem-se os maiores valores de $\mathrm{PI}_{\text {máx }}$ à rapidez e eficácia exigidas durante a intensa captação de ar para a produção do som. ${ }^{1}$

A pressão das vias aéreas e a proporção de fluxo de ar se diversificam de acordo com o tipo de instrumento de sopro ${ }^{13}$ resultando em diferentes volumes pulmonares e graus de força da musculatura respiratória. ${ }^{1,14} \mathrm{~A}$ execução exata do som implica ter habilidades respiratórias precisas. Os altos volumes pulmonares, por sua vez, também derivam de um longo período de treinamento. Igualmente, o tom da música, duração do tom e o tipo de instrumento de sopro exercem influência sobre o recrutamento de músculos respiratórios específicos. ${ }^{15}$ Nesse sentido, com a melhora da mecânica respiratória devido ao aumento da capacidade, pode gerar maiores volumes pulmonares após essa intervenção com a flauta e promover saúde e qualidade de vida na longevidade. ${ }^{16}$

As atividades lúdicas proporcionam resultados positivos na saúde de idosos, como pode ser observado em alguns estudos. ${ }^{17} \mathrm{O}$ mesmo pode ser encontrado no presente estudo, em que foi realizada intervenção com a flauta que se trata também de uma atividade lúdica e foi notada melhora na força muscular respiratória de idosas. Essas práticas estimulam diversos sentidos ao mesmo tempo, melhorando a socialização, comunicação e a qualidade de vida dos participantes. ${ }^{18-19}$

Assim, um dos maiores desafios encontrados na promoção da saúde é prevenir e retardar as alterações decorrentes ao envelhecimento, exigindo uma atenção maior em intervenções que promovam benefícios 
para essa população, promovendo um envelhecimento mais ativo e saudável. ${ }^{20}$ Após os achados deste estudo, ele apresenta mais uma oportunidade de promover a manutenção da saúde na senescência.

Nesse sentido, as UNATI vêm sendo uma boa oportunidade de espaços para desenvolver práticas de promoção à saúde, pois elas apresentam uma abordagem multidisciplinar no currículo e na metodologia de trabalho, priorizando o processo de valorização da terceira idade analisando constantemente os diversos aspectos: biológico, psicológico, político, espiritual e sociocultural, com objetivo de integrar esses cidadãos à sociedade contemporânea, tornando-os mais ativos, alegres e participativos com qualidade de vida..$^{21-22}$

A carência de estudos no âmbito dos instrumentos musicais como jogos lúdicos em idosos na melhora da força muscular respiratória limitação do presente estudo, pois o público idoso, o qual apresenta peculiaridades e respostas fisiológicas distintas decorrentes do processo de envelhecimento. Além disso, a diversidade metodológica em- pregada nos estudos (tipo de instrumento de sopro, tempo de prática e escassez de estudos de intervenção) ${ }^{16}$ dificultou a comparação com os achados deste estudo. Outras limitações estiveram neste estudo, como a ausência de um grupo controle, o reduzido tamanho amostral e a ausência de idosos do sexo masculino.

Este estudo forneceu importantes contribuições para a sociedade em relação à flauta por ser uma atividade simples, com recurso de fácil acesso e baixo custo, que pode ser utilizado em programas de promoção da saúde com a terceira idade.

\section{CONSIDERAÇões FINAIS}

Diante dos resultados apresentados, conclui-se que houve aumento da FMR de idosas submetidas a um treinamento com flauta doce, as quais nunca tocaram qualquer instrumento de sopro. Nesse sentido, faz-se necessária a condução de novas investigações que elucidem a temática de ganho da FMR em idosos, tendo como estratégia a prática de instrumentos de sopro.

\section{REFERÊNCIAS}

1 Ferreira CAS, Isern MRM, Baroni CCA, Carrocini VK. Análise da função pulmonar em músicos que tocam instrumento de sopro. O Mundo da Saúde. 2010; 34 (2): 200-9.

2 Barcellos FB, Diniz LN. Programa de Extensão Doce Flauta de Pelotas: Descrição, Andamento e Propostas. Arte e Educação. 2012; 1 (23): 496-499.

3. Meira EC, Sena ELS, Souza AS, Coronago VMMO, Gonçalves LHT, Santos ET, et al. Tecnologia Assistiva de Vivências Musicais na recuperação vocal de idosos portadores de Doença de Parkinson. Revista Brasileira de Geriatria e Gerontologia. 2008; 11 (3): 341-355.

4 Fiz JA, Aguilar J., Carreras A. et al. Maximum respiratory pressures in trumpet players. Chest. 1993; 104: 1.203-4.

5 Azevedo JVV, Santos CA, Silva MT, Olinda RA, Santos DAS. Análise das variações climáticas na ocorrência de doenças respiratórias por influenza em idosos na região metropolitana de João Pessoa-PB. Revista Sociedade \& Natureza. 2017; (1): 123-135. 
6 Queiroz RS, Carneiro JAO, Fagundes LC, Brito TA, Coqueiro RS, Fernandes MH. A relação VEF1/CVF não é preditora da síndrome da fragilidade em idosos. Revista de enfermagem UFPE. 2017; 11 (4): 1.779-1.783.

7 Simões RP, Deus APL, Auad MA, Dionísio J., Mazzonetto M., Borghi-Silva A. Maximal respiratory pressure in healthy 20 to 89 year-old sedentary individuals of central São Paulo State. Revista Brasileira de Fisioterapia. 2010; 14 (1): 60-7.

8 Rocha FR, Brüggemann AKV, Francisco DDS, Medeiros CSD, Rosal D., Paulin E. Diaphragmatic mobility: relationship with lung function, respiratory muscle strength, dyspnea, and physical activity in daily life in patients with COPD. Jornal Brasileiro de Pneumologia. 2017; 43 (1): 32-37.

9 Pascotini FS, Ramos MC, Silva AMV, Trevisan ME. Espirometria de incentivo a volume versus a fluxo sobre parâmetros respiratórios em idosos. Fisioterapia e Pesquisa. 2013; 20 (4): 355-360.

10 Fonseca M., Cader S., Matheus S., Dantas E. Efeitos de programas de treinamento muscular respiratório na força muscular respiratória e na autonomia funcional de idosos. Memorialidades. 2017; 13 (25): 89-118.

11 Cossette I. Mécanique respiratoire des flûtistes professionnels. Revista Malasia Respiratory. 2002; 19: 197-206.

12 Kock KS, Ruckhaber SL. Utilização de instrumento de sopro como complemento terapêutico para pacientes pneumopatas crônicos. Movimenta. 2012; 5 (3): 266-72.

13 Kreuter M., Kreuter C., Herth F. Pneumological Aspects of Wind Instrument Performance Physiological, Pathophysiological and Therapeutic Considerations. Pneumologie. 2008; 62 (2): 83-87.

14 Dhule SS, Sunita BN, Gawali SR. Pulmonary Function Tests in Wind Instrument Players. International Journal of Science and Research. 2013; 2 (5): 384-6.

15 Cossette I., Monaco P., Aliverti A., Mackem P: Chest wall dynamics and respiratory muscle recruitment during professional flute playing. Respiratory Physiology \& Neurobiology. 2008; 160: 187-95.

16 Fonseca MDA, Cader SA, Dantas EHM, Bacelar SC, Silva EBD, Leal SMDO. Respiratory muscle training programs: impact on the functional autonomy of the elderly. Revista da Associacao Medica Brasileira. 2010; 56 (6): 642-648.

17 Cyrino RS, Silva LED, Souza MR, Lara CJB, Pereira TS. Atividades lúdicas como estratégia de educação em saúde com idosos. Revista de Ciência e Extensão. 2016; 12 (3): 154-163.

18 Oliveira CS, Costa SRR, Santos ICL, Lemos CES. Oficina de educação, memória, esquecimento e jogos lúdicos para a terceira idade. Revista Ciência e Extensão. 2012; 8 (1): 8-17.

19 Fernandes APS, Oliveira RC. O idoso e a ludicidade. Revista de Ciências Humanas, Linguagem, Letras e Artes. 2012; 20 (2): 151-160.

20 Veras RP. Experiências e tendências internacionais de modelos de cuidado para com o idoso. Ciência \& Saúde Coletiva. 2012; 17 (1): 231-238.

21 Rodrigues JP, Fernandes CAM. Lazer e Sociabilidade: Sobre as Memórias e Motivações de Idosos Participantes de uma Experiência de Educação Não Formal na Zona Leste da Cidade de São Paulo. Revista do Programa de Pós-graduação Interdisciplinar em Estudos do Lazer. 2017; 19 (4): 201-225.

22 Lolli MCGS, Lolli LF, Maio ER. Universidade aberta à terceira idade: uma tentativa de emancipação. Revista Labor. 2017; 1 (12): 131-151. 


\section{DAdos DOS AUTORES}

\section{Dayane Aparecida Viana}

Mestre em Atenção à Saúde pela Universidade Federal do Triângulo Mineiro (UFTM). Doutoranda em Gerontologia pela Universidade Estadual de Campinas (UNICAMP). Fisioterapeuta. Campinas/SP - Brasil. dayane.viana@hotmail.com

\section{DANiel Vicentini de Oliveira}

Mestre em Promoção da Saúde pelo Centro Universitário Cesumar (UNICESUMAR). Doutor em Gerontologia pela Universidade Estadual de Campinas (UNICAMP). Fisioterapeuta e Profissional de Educação Física. Maringá/PR - Brasil. d.vicentini@hotmail.com

\section{Maycon Sousa Pegorari}

Mestre e Doutor em Atenção à Saúde pela Universidade Federal do Triângulo Mineiro (UFTM). Fisioterapeuta. Macapá/AP - Brasil. mayconpegorari@yahoo.com.br

\section{Lilane Maria Alves Silva}

Mestre e Doutoranda em Atenção à Saúde pela Universidade Federal do Triângulo Mineiro (UFTM). Fisioterapeuta. Uberaba/MG - Brasil. lilanealves@yahoo.com.br

\section{GuAlberto RuAs}

Mestre e Doutor em Fisioterapia pela Universidade Federal de São Carlos (UFSCAR). Fisioterapeuta. Uberaba/MG - Brasil - gualbertoruas@yahoo.com.br

\section{Lislei Jorge Patrizzi}

Mestre em Reabilitação pela Universidade Federal de São Paulo (UNIFESP). Doutora em Clinica Médica pela Universidade de São Paulo (USP). Fisioterapeuta. Uberaba/MG - Brasil. lispatrizzi@gmail.com

\section{JUREMA Ribeiro Luiz GonÇALVES}

Mestre e Doutora em Enfermagem pela Escola de Enfermagem de Ribeirão Preto da Universidade de São Paulo (EERP-USP). Enfermeira. Ribeirão Preto/SP - Brasil. jugoncalves@ enfermagem.uftm.edu.br

Submetido em: 13-3-2018

Aceito em: 7-5-2019 